\title{
High Dexterity Snake-Like Robotic Slaves for Minimally Invasive Telesurgery of the Upper Airway
}

\author{
Nabil Simaan ${ }^{1}$, Russell Taylor ${ }^{1}$, and Paul Flint ${ }^{2}$ \\ ${ }^{1}$ Department of Computer Science \\ NSF Engineering Research Center for \\ Computer-Integrated Surgical Systems and Technology \\ The Johns Hopkins University \\ 3400 North Charles Street - NEB B26 \#7, Baltimore, Maryland, 21218 \\ \{nsimaan, rht\}@es.jhu.edu \\ http://www.cisst.org \\ ${ }^{2}$ Department of Otolaryngology - Head \& Neck Surgery \\ Johns Hopkins School of Medicine \\ 601 North Caroline Street, Baltimore, Maryland, 21287 \\ pflint@jhmi.edu \\ http: / / www.hopkinsmedicine.org/otolaryngology
}

\begin{abstract}
This paper reports our efforts to develop an integrated system for telesurgery of the throat and upper airway. The system is described while focusing on the novel design of its slave robot. The slave robot is a 3-armed robot that implements novel Distal Dexterity Units (DDU's). These DDU's are dexterous robots for surgical tool manipulation and suturing in confined spaces. Each DDU is composed from a Snake-Like Unit (SLU) and a detachable Parallel Manipulation unit (PMU). The proposed design of the DDU's provides an enhanced downsize scalability and distal dexterity that are crucial for medical applications such as laryngeal surgery where simultaneous manipulation of 2-3 long tools through a narrow laryngeoscope is required. The paper presents the design of these units and the experimentation results with a $4.2 \mathrm{~mm}$ diameter SLU to be used for constructing the first DDU for the 3-armed slave robot.
\end{abstract}

\section{Introduction}

Previous work on telerobotic systems for MIS (Minimally Invasive Surgery) has focused on endoscopic surgery of the chest and abdomen while using several different mechanical architectures for slave robots. These mechanical architectures included remote-center-of-motion mechanisms (e.g., [1, 2]), serial-link robots with passive joints [3], and mini-parallel robots (e.g., $[4,5]$ ). A recent survey of these works has been presented in [6]. A fundamental challenge for these robots is the kinematic constraint imposed by the passage of the surgical tools through fixed entry ports into the 
patient's body. If more than four Degrees-of-Freedom (DoF) are required in manipulating a surgical instrument, then some form of distal dexterity mechanism is required.

The purpose of this work is to develop a system for MIS of the upper airway including the throat and larynx - an unusually challenging task due to the shape of the airway and position of the larynx, the need to perform complex procedures on or beyond the vocal folds, and the need to simultaneously manipulate 2-3 long instruments through a predetermined entry port. This aim includes developing down-sizable Distal Dexterity Units (DDU's) for precise surgical tool manipulation in confined spaces where the required diameter of these units is less than $4.5 \mathrm{~mm}$.

\section{Clinical Relevance, Setup, and Requirements}

The upper airway is a long, narrow, and irregularly shaped organ that includes the pharynx (throat), hypopharynx, and larynx, commonly referred to as the voice box. These areas are subject to a variety of benign and malignant growths, paralysis, and scar tissue formation requiring surgical interventions for excision and/or reconstruction. These procedures (e.g. partial or total laryngectomy, vocal fold repositioning, and laryngotracheal reconstruction) are routinely performed using open surgical techniques on the expense of damaging the integrity of the framework supporting the laryngeal cartilage, muscle, and the connective tissue vital to normal function. Minimally invasive endoscopic procedures are generally preferred over open procedures, thereby, preserving laryngeal framework integrity, promoting faster recovery and frequently overcoming the need for tracheostomy.

Figure 1 shows a typical MIS setup for laryngeal surgery. The internal regions of the airway are accessed by using an array of long instruments (usually ranging between 240 to $350 \mathrm{~mm}$ long) through a laryngoscope that is inserted into the patient's mouth and serves as a visualization tool and a

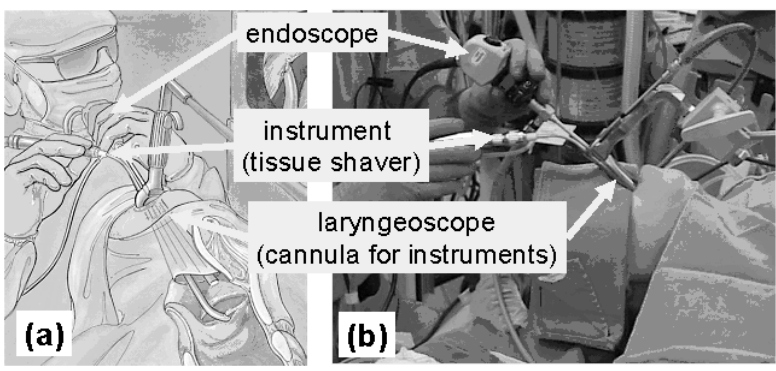

Fig. 1. A typical surgical setup for MIS of the throat (a) artist rendering (b) actual surgical setup guide for surgical instrumentation. The laryngeoscope is typically $180 \mathrm{~mm}$ long with an oval cross-section usually ranging between $16-20 \mathrm{~mm}$ in width at its smallest cross section. This surgical setup requires the surgeon to manipulate several long tools (for example one tool for suction and another for tissue manipulation) that are constrained to 4 DoF motions and lacking tool-tip dexterity. For these limitations, laryngeal MIS is currently limited to simple operations such as microflap elevation, excisional biopsies, and removal of papilloma using laser or powered microdebrider. Functional reconstructive procedures (e.g. tissue flap rotation or suturing), are not performed in throat MIS; although, re- 
construction of the vocal fold structures as accurately as possible is crucial for maintaining the voice characteristics. Suture closure of surgical defects has been shown to reduce scar tissue, shorten healing time, and result in improved laryngeal function and sound production $[7,8]$. This seemingly simple operation is very difficult, if not impossible, to perform in laryngeal MIS. This work addresses these needs by developing a system to allow surgeons to perform complex functional reconstruction tasks and suturing in MIS of the upper airway.

We use suturing to define the required workspace and force application capability of our system. The goal reachable workspace is a cylindrical work volume about 40 $\mathrm{mm}$ in diameter and $50 \mathrm{~mm}$ in height and located $180-250 \mathrm{~mm}$ axially down the throat. The Distal Dexterity Units (DDU's) should be able to bend $90^{\circ}$ sideways in any direction of while maintaining their ability to apply 1 Newton at its tip for tissue manipulation/suturing purposes.

\section{Snake-Like Robots for Dexterity Enhancement in MIS}

Several approaches to distal tool dexterity enhancement have been reported. Many systems (e.g.,[2]) use wire actuated articulated wrists. Other systems use snake-like active bending devices. For example, [9] used bending SMA (Shape Memory Alloy) forceps for laparoscopic surgery. Dario et al [10] presented an SMA actuated 1 DoF planar bending snake device for knee arthroscopy and Reynaerts [11] designed a hyper-redundant SMA actuated snake for gastro-intestinal intervention. Recently, Piers et al. [12] presented a two DoF $5 \mathrm{~mm}$ diameter wire-driven snake-like tool using super-elastic NiTi flexure joints and Ikuta et al. [13] reported a $\varnothing 3 \mathrm{~mm}$ wire-actuated articulated robot attached at the tip of a flexible stem for microsurgery inside deep and narrow spaces.

Cavusoglu et al. [14] analyzed alternative designs of a 3 DoF wrist for MIS suturing. They proposed a method to determine the workspace and to optimize the position of the entry port in the patient's body to provide optimal dexterity. Faraz and Payendeh [15] recently analyzed three architectures of endoscopic wrists: a simple wire actuated joint, a multi-revolute joint wrist, a tendon snake-like wrist. They compared these joints in terms of dexterity and showed the superiority of the snake-like wrist over the other two wrists in terms of dexterity.

In chest and abdomen MIS, the entry portals for surgical instruments are usually placed some distance apart, and the instruments approach the operative site from somewhat differing directions. This makes it possible (though sometimes inconvenient and limiting) for telesurgical systems such as the DaVinci or Zeus use rather large robotic slave manipulators for extracorporeal instrument positioning. The optimal placement of entry portals based on dexterity requirements for particular procedures is an important subject and has recently been addressed by several authors including Adhami et al. [16] and Cannon et al. [17]. In MIS of the throat, the entry port is predetermined and no such optimization is possible. The slave robot presented in the next section is designed to answer this limitation. 


\section{The Design of the 3-Armed Slave}

Fig. 2 presents the system we are currently developing for throat MIS, [18]. This system has the same master-slave architecture as the DaVinci ${ }^{\circledR}[2]$ or any other telesurgical robot. Although this system is specialized for throat surgery, some components (such as the DDU's) are suitable for minimally invasive microsurgery in confined spaces in general. This paper will focus on presenting the slave robot of Fig. 3.

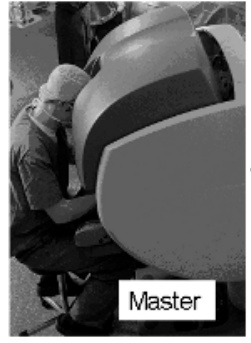

(a)

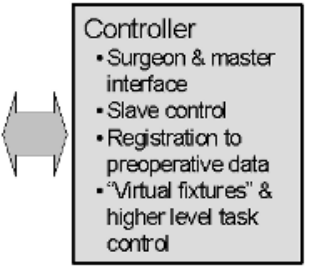

(b)

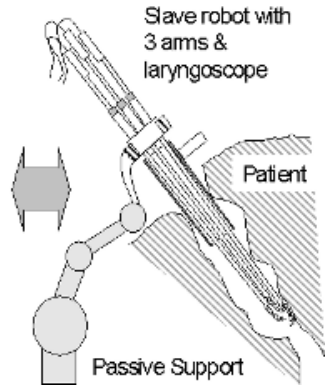

(c)

Fig. 2. System overview: (a) a master interface with force and visual feedback (b) a smart controller used for master-slave integration and control of the redundant degrees of freedom offered by the slave robot (c) a 3-armed slave robot for simultaneous manipulation of 2-3 robotic arms equipped with distal dexterity units

The slave robot is a three-armed robot working through a laryngeoscope, Fig. 2-(c). Our design includes a laryngoscope, a base link, two similar DDU's for tool/tissue manipulation, and another DDU for suction. Each DDU is a 5 DoF robot mounted on a corresponding DDU holder, which is manipulated by a corresponding $4 \mathrm{DoF}$ tool manipulation unit (TMU) that controls the angle of approach, the rotation about and the position along

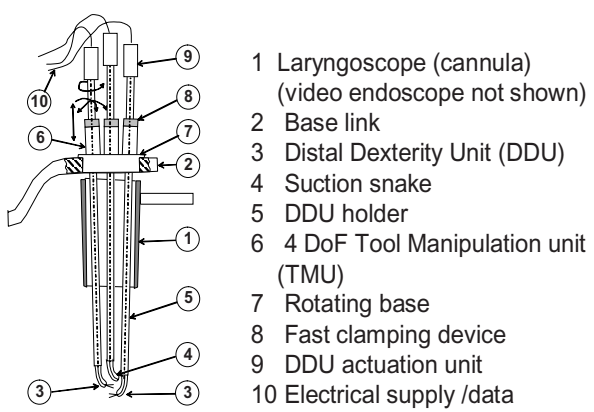

Fig. 3. The 3-armed slave robot the axis of the DDU holder. The TMU's are mounted on a rotating base unit (RBU) permitting the system to be oriented within the throat so as to minimize collisions between DDU holders. The DDU holders are thin tubes (about $4 \mathrm{~mm}$ in outside diameter) providing an actuation pathway for the DDU and possibly a light-source or a suction channel. Each TMU is equipped with a fast clamping device for adjusting the axial location of the DDU. The actuation unit of each DDU is located at its upper extremity and the actuation is by super-elastic tubes operated in push-pull mode.

This system implements actuation redundancy in the design and control of the DDU's. Each DDU has 7 actuated joints and each TMU has 4 DoF. The total number of actuated joints for the slave is 34 . 
The DDU (Distal Dexterity Unit). The DDU is composed from a snake-like unit and a detachable parallel manipulation unit attached at its tip, Fig. 4. It is designed to bypass obstacles (such as the vocal folds) and to perform suturing by transforming the rotation of the DDU holder about its axis into rotation about its backbone axis.

The multi-backbone snake-like unit. The SLU of Fig. 4-(a) is a 2 DoF robot composed from a base disk, an end disk, several spacer disks, and four super-elastic NiTi tubes. These tubes are called the backbones of this SLU. The central tube is the primary backbone while the remaining three tubes are the secondary backbones. The secondary backbones are equidistant from the central backbone and from one another. The central backbone is attached to both the base and end disks and to all spacer disks while the secondary backbones are attached only to the end disk and are free to slide and bend through properly dimensioned holes in the base and spacer disks. These secondary backbones are used for actuating this snake-like device and they pass through guiding channels in the DDU holder to allow their actuation in both push and pull modes. The spacer disks prevent buckling of the central and secondary backbones and keep an equal distance between them.

\begin{tabular}{|c|l|}
\hline 1 & gripper \\
\hline 2 & $\begin{array}{l}\text { moving } \\
\text { platform }\end{array}$ \\
\hline 3 & $\begin{array}{l}\text { parallel } \\
\text { stage wires }\end{array}$ \\
\hline 4 & gripper wire \\
\hline 5 & end disk \\
\hline 6 & spacer disk \\
\hline 7 & $\begin{array}{l}\text { central } \\
\text { backbone }\end{array}$ \\
\hline 8 & base disk \\
\hline 9 & $\begin{array}{l}\text { DDU } \\
\text { holder }\end{array}$ \\
\hline
\end{tabular}

Fig. 4. (a) The DDU (Distal Dexterity Unit) is composed from a snake-like unit and a detachable parallel manipulation unit (b) detachable parallel manipulation unit for precise wrist-like and axial motions; and surgical tool interchangeability

This design has several advantages over standard designs using discrete backbones (articulated serial chains). By using flexible backbones, the dependency on small universal joints and wires is removed. This reduces manufacturing costs and enhances downsize scalability. The use of tubes for the backbones provides a secondary application for them as suction channels, actuation channels for the tool mounted on the SLU distal end or as a source of light for imaging. By using three push-pull secondary backbones for actuation, it is possible to satisfy the statics of the structure while preventing buckling of the backbones as was proposed in [18]. This further enhances the downsize scalability while maintaining the force application capability of these SLU's on a level large enough for tool manipulation for delicate tasks (1-2 Newton).

The detachable milli-parallel unit. The SLU is capable bending sideways in any direction, hence, providing 2 DoF for distal dexterity. To enhance it with surgical tool interchangeability and additional precise wrist action and axial motion, we are currently designing and constructing the detachable parallel unit of Fig. 4-(b). The paral- 
lel mechanical architecture has been chosen for providing precise and high-accuracy motions in a small workspace while utilizing its inherent rigidity $[4,5]$.

The detachable milli-parallel unit is constructed from super-elastic actuation wires passing through the secondary backbones, spherical joints, and a moving platform to which a gripper of a tool is affixed, Fig. 4 -(b). The moving platform is machined with matching groves such that the balls attached to the end of the actuation wires match its diameter and a flexible locking ring is placed around the circumference of the moving platform to maintain these balls inside their grooves. To detach the gripper/tool one removes the lock ring and removes the moving platform.

There are two possible operation modes using the detachable milli-parallel unit. In the first operation mode, the actuation wires are used only to extend axially in order to attach a new moving platform equipped with another tool. Once the tool is attached, then the actuation wires are retrieved until the moving platform is secured on the end platform of the SLU. In the other mode, for operations requiring small workspace and fine motions, the actuation wires are used to actuate the moving platform as a three DoF parallel platform with flexible extensible links.

We are also considering another alternative design for this parallel manipulation unit for small-diameter DDU's. This design sacrifices detachability for downsize scalability by eliminating the spherical joints and relying completely on the flexibility of the superelastic links manipulating the moving platform.

\section{Prototype Design and Construction}

Using the kinematic and static formulation we presented in [18], the action of the SLU was simulated for designing a $\varnothing 4 \mathrm{~mm}$ SLU shown in Fig. 5-(a) in several configurations inside its workspace. Fig. 5-(b) shows the prototype we constructed based on this design. This prototype is $4.2 \mathrm{~mm}$ in diameter, $28 \mathrm{~mm}$ long, and it uses $\varnothing 0.66 \mathrm{~mm}$ superelastic backbones for the primary and secondary backbones. In our experiment we manually actuated only two out of three secondary backbones. The unit is able to bend more than $70^{\circ}$ sideways in any direction while applying forces at its tip larger than 1 Newton. Once the actuation unit is constructed, we will implement

(a)

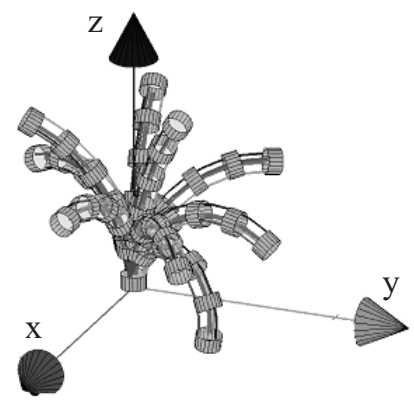

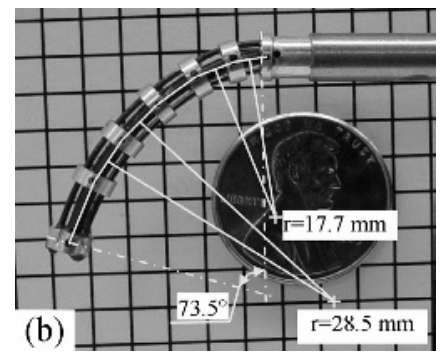

Fig. 5. Snake like unit: (a) simulation for design purposes (b) the $\varnothing 4.2 \mathrm{~mm}$ snake-like prototype based on the parameters of the simulation in (a) 
actuation redundancy to actuate all three secondary backbones in push-pull operation. This will help bend the SLU in larger bending angles while keeping the strain in the backbones within a $4 \%$ limit for guarding against degradation of the backbones' superelastic properties in repeated operation. The figure shows the total bending angle and the radiuses of curvature of the primary backbone along two different sections. This non-constant radius of curvature is due to the elongation of the secondary backbones due to actuation forces and due to the manufacturing tolerances of the disks. The variable spacing of the disks was used to reduce this effect and to prevent buckling at the base of the SLU that is shown doing spatial motion in Fig. 6.
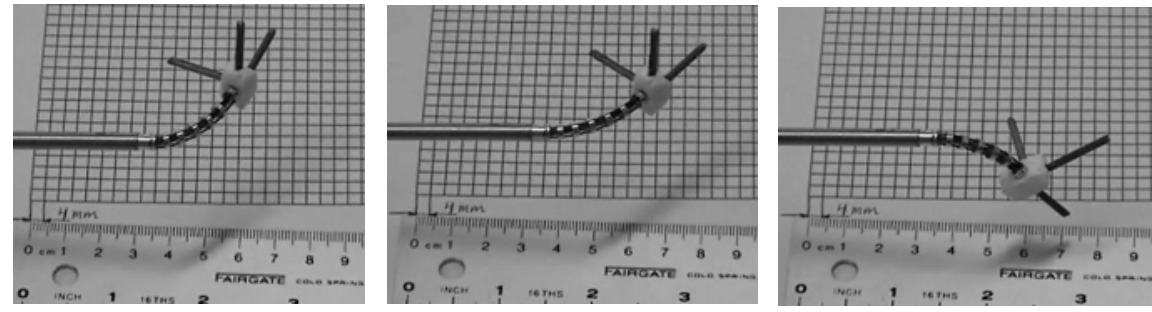

Fig. 6. The snake-like unit of Fig. 5-(b) performing spatial motions. This unit was able to apply more than 1 Newton at its tip and to bend more than $70^{\circ}$ sideways in any direction by actuating only two out of its three available secondary backbones

\section{Conclusions}

This paper presented our work on designing a high dexterity, high accuracy slave for tele-operated MIS of the throat and upper airway. This clinical application was discussed and the benefits of distal dexterity enhancement for functional reconstruction of tissue and suturing inside the larynx were highlighted. These seemingly simple operations are currently extremely difficult to perform in a MIS.

Although this system is not yet finalized and clinically demonstrated, we believe it includes two components that will enhance throat MIS in particular and microsurgery in general. These components are the $34 \mathrm{DoF}$ three-armed slave robot capable of manipulating through a narrow laryngeoscope and the novel Distal Dexterity Units (DDU's) that are useful for precise dexterous operations in small confined spaces. Each DDU is a dexterous robot composed of a SLU and a detachable parallel manipulation unit - all designed to enhance downsize scalability to diameters smaller than $5 \mathrm{~mm}$. The design of this SLU implements several flexible tubular backbones. This allows it to be used for visualization purposes, aspiration, micro drilling and to be easily downsized to diameters smaller than $4 \mathrm{~mm}$. Finally, we presented our first prototype of a $\varnothing 4.2 \mathrm{~mm}$ SLU. It was shown to have the ability to exert forces larger than 1 Newton at its tip and to bend more than $70^{\circ}$ in any direction.

Acknowledgments. This work was partially funded by the National Science Foundation (NSF) under Engineering Research Center grant \#EEC9731478, NSF grant \#IIS9801684, and by the Johns Hopkins University internal funds. 


\section{References}

1. R. Taylor, J. Funda, B. Eldridge, S. Gomory, K. Gurben, D. LaRose, M. Talamini, L. Kavoussi, and J. Anderson, "A Telerobotics Assistant for Laparoscopic Surgery," IEEE Engineering in Medicine and Biology Magazine, vol. 14, pp. 279-288, 1995.

2. G. Guthart and K. Salisbury, "The Intuitive ${ }^{\mathrm{TM}}$ Telesurgery System: Overview and Application," IEEE International Conference on Robotics and Automation, pp. 618-621, 2000.

3. J. M. Sackier and Y. Wang, "Robotically assisted laparoscopic surgery from concept to development," Surgical Endoscopy, vol. 8, pp. 63-66, 1994.

4. N. Simaan and M. Shoham, "Robot Construction for Surgical Applications," The 1st IFAC Conference on Mechatronic Systems, Darmstadt, Germany, pp. 553-558, 2000.

5. M. Shoham, M. Burman, E. Zehavi, L. Joskowicz, E. Batkilin, and Y. Kunicher, "BoneMounted Miniature Robot for Surgical Procedures: Concept and Clinical Applications," IEEE Transactions on Robotics and Automation, vol. 19, pp. 893-901, 2003.

6. R. Taylor and D. Stianovici, "Medical Robotics in Computer-Integrated Surgery," IEEE Transactions on Robotics and Automation, vol. 19, pp. 765-781, 2003.

7. D. J. Fleming, S. McGuff, and C. B. Simpson, "Comparison of Microflap Healing Outcomes with Traditional and Microsuturing Techniques: Initial Results in a Canine Model," Ann Otol Rhinol Laryngol., vol. 110, pp. 707-712, 2001.

8. P. Woo, J. Casper, B. Griffin, R. Colton, and C. Brewer, "Endoscopic Microsuture Repair of Vocal Fold Defects," J. Voice, vol. 9, pp. 332-339, 1995.

9. Y. Nakamura, A. Matsui, T. Saito, and K. Yoshimoto, "Shape-Memory-Alloy Active Forceps for Laparoscopic Surgery," IEEE International Conference on Robotics and Automation, pp. 2320-2327, 1995.

10. P. Dario, C. Paggetti, N. Troisfontaine, E. Papa, T. Ciucci, M. C. Carrozza, and M. Marcacci, "A Miniature Steerable End-Effector for Application in an Integrated System for Computer-Assisted Arthroscopy," IEEE International Conference on Robotics and Automation, pp. 1573-1579, 1997.

11. D. Reynaerts, J. Peirs, and H. Van Brussel, "Shape memory micro-actuation for a gastrointesteinal intervention system," Sensors and Actuators, vol. 77, pp. 157-166, 1999.

12. J. Piers, D. Reynaerts, H. Van Brussel, G. De Gersem, and H. T. Tang, "Design of an Advanced Tool Guiding System for Robotic Surgery," IEEE International Conference on Robotics and Automation, pp. 2651-2656, 2003.

13. K. Ikuta, K. Yamamoto, and K. Sasaki, "Development of Remote Microsurgery Robot and New Surgical Procedure for Deep and Narrow Space," IEEE International Conference on Robotics and Automation, pp.1103-1108, 2003.

14. M. Cavusoglu, I. Villanueva, and F. Tendick, "Workspace Analysis of Robotics Manipulators for a Teleoperated Suturing Task," IEEE/RSJ International Conference on Intelligent Robots and Systems, Maui, H1, pp. 2234-2239, 2001.

15. A. Faraz and S. Payandeh, "Synthesis and Workspace Study of Endoscopic Extenders with Flexible Stem," (online report) Simon Fraser University, Canada 2003.

16. L. Adhami and E. C. Maniere, "Optimal Planning for Minimally Invasive Surgical Robots," IEEE Transactions on Robotics and Automation, vol. 19, pp. 854-863, 2003.

17. J. W. Cannon, J. A. Stoll, S. D. Sehla, P. E. Dupont, R. D. Howe, and D. F. Torchina, "Port Placement Planning in Robot-Assisted Coronary Artery Bypass," IEEE transactions on Robotics and Automation, vol. 19, pp. 912-917, 2003.

18. N. Simaan, R. Taylor, and P. Flint, "A Dexterous System for Laryngeal Surgery - MultiBackbone Bending Snake-like Slaves for Teleoperated Dexterous Surgical Tool Manipulation," IEEE International Conference on Robotics and Automation, New Orleans, pp.351$357,2004$. 